\title{
A cross-cultural study of icons and images used in North American web design
}

\author{
E. Knight ${ }^{1}$, C. Gunawardena ${ }^{2}$, F. Bouachrine ${ }^{3}$, N. Dassanayake ${ }^{4}$, \\ T. Gnanakumar ${ }^{5} \&$ C. Kulasuriya ${ }^{6}$ \\ ${ }^{1}$ University of New Mexico, USA \\ ${ }^{2}$ Department of Organizational Learning and Instructional Technologies, \\ University of New Mexico, USA \\ ${ }^{3}$ Al-Akhawayn University in Ifrane, Morocco \\ ${ }^{4}$ Open University of Sri Lanka, Sri Lanka \\ ${ }^{5}$ Open University of Sri Lanka, Sri Lanka \\ ${ }^{6}$ Open University of Sri Lanka, Sri Lanka
}

\begin{abstract}
This paper reports the results of a study which examined differences in perceptions of the meaning of icons and images drawn from US academic web sites. To provide a cross-cultural perspective, the study was conducted in the USA at the University of New Mexico; in Morocco at Al-Akhawayn University in Ifrane; and in Nawala, Nugegoda at the Open University of Sri Lanka.

A questionnaire containing 18 icons and images drawn from 26 US academic websites was distributed via the Internet. Participants were asked to assign meanings to each icon or image and to select a preferred image to represent group discussion online, Internet chat, submitting an assignment online, and accessing a library. In addition, participants were asked to choose from three images of professors which they would prefer to take a course from and why.

Differences in the interpretation of meanings and preferences for specific icons and images are related to the cultural perspectives of the participants. Implications and recommendations for the design of icons and images for use in cross-cultural online learning environments are discussed.

Keywords: web design, design of icons and images, multimedia design, online learning environments, cultural differences, cross-cultural design.
\end{abstract}




\section{Introduction}

Icons have been defined as graphic symbols in the form of concrete pictures which represent real or imagined objects Tzeng et al. [1]. Other definitions have emphasized the use of icons to represent objects, actions, applications, utilities and commands within a computer system Preece [2]. Incorporated in online learning environments, icons enhance the learner's ability to use and control the capabilities available within the environment. An icon may describe the kind of information available, the nature of specific functions, which can be performed, or activities that may be accessed. If the meaning of a particular icon or image is not clear, the learner's ability and motivation to interact within the environment will be directly impeded.

Because of the constraints of its physical form and the need to convey a visual meaning, which is relatively predictable across a diverse group of learners, the icon is one of the most challenging of design problems. In terms of the design process, the decision regarding the selection of a referent or metaphor which can effectively represent a particular computer function, must precede any graphic solution. An understanding of how icons are interpreted is necessary to this aspect of the design process. Graphic designers know that interpretations of the meaning of an effectively designed icon occur within a predicable range Jonesson et al. [3]. The narrowness of the interpretation range is a valid indication of the effectiveness of the icon.

To provide a context for this study, the following areas from which factors related to the design and use of icons have arisen are identified. The first relates to the globalization of the user interface and cultural differences in the perception and understanding of interface design Evers [4]. The second area focuses on classes of icons that differ in terms of their functions and the attributes associated with how they communicate graphically Jonesson et al. [3]. The third area relates to sources of cultural differences influencing the interpretation of icons and images and how dimensions of culture identified as sensitive are related to icon and interface design Hofstede [5].

Localization of software is becoming more important in the global market place Fernandes [6]. As a result, there has been an increase in the prevalence of culturally biased products released in different languages without respect for the appropriateness of the icons or images in regard to representing their intended meaning in the target culture Evers [4]. Yeo [7] distinguishes between overt and covert factors that need to be addressed in order for software to be adapted for a specific culture. Culturally driven reactions to covert elements will vary, depending on whether the message conveyed is understood in the target culture Yeo [7]. Some messages may not be within the common cultural experience of a group and should therefore be changed or discarded.

The differentiation of icons into classes has been based largely on the reference to which the icon relates. Jonesson et al. [3] differentiate between icons that describe the kind of information the user will access as a result of clicking the icon and those that describe the function to which the icon will provide access. Further classifications have been based on the representation's degree of 
realism or abstraction. Preece [2] distinguishes three types of metaphors commonly used in interface design: verbal metaphors, virtual interface metaphors, and composite metaphors. The comprehension of such metaphors is dependent on the users previous experience and culture and would occur in the reasoning phase of perception Evers [4]. Findings by Duncker [8] indicated that experience acquired in a cultural context using a referent object is necessary in order for a metaphor to be appropriate, i.e. to match the user's mental model.

Hofstede [5] identified sources of cultural differences relating to specific patterns of thinking, feeling, and acting as well as differences related to the amount of information an individual is comfortable receiving. Cultural dimensions identified by Hofstede [5] include: Power Distance, IndividualismCollectivism, Uncertainty Avoidance, Masculinity-Femininity, and Long-Term Orientation. Additional sources of difference suggested by Shaw and BarrettPower [9] include readily detectable attributes which represent cultural values, perspective, attitudes and beliefs, and those described as underlying attributes which are of a personal nature, including socioeconomic and personal status, education, functional specialization, etc.

\section{Methods}

\subsection{Purpose}

The purpose of this study was to examine from a cross-cultural perspective, the effectiveness of specific icons in representing online learning functions, actions and information types as indicated by the range and emphasis of the interpretations of the icons as visual representations only, or as signifying functions, actions or other objects. It was an hypothesis that the frequency of Internet use and familiarity with the online activities and information types would determine the participant's ability to interpret the intended meanings of the icons and images, and that individual preferences for the images and icons in representing specific ideas and functions would be related to the user' cultural values.

\subsection{Participants}

Researchers at the three institutions solicited participants from the respective campuses as well as from off-campus locations. Fifty-three participants from Morocco, sixty-eight from Sri Lanka, and fifty-eight from the US completed the questionnaire. English is taught as a second language in the Sri Lankan schools and is widely spoken by the educated. Those who use the Internet are fluent in English. Among Sri Lankans, the fluency level in English was much higher than for Moroccans who tend to be more fluent in French. Within the Middle Atlas region of Morocco, data was collected from four areas: Ifrane (19\%) where the University is located and students are primarily from other regions of Morocco, representing the upper middle class or the rich. AinLeuh (21\%) a depressed, predominantly, Berber small town/village in a very rural location; 
Azrou (28\%) a mid sized predominantly Berber town close to Ifrane which had a mixed clientele of people; and

Fez Medina (21\%) the more traditional, more conservative part of the big city Fez. 1. Other areas (11\%) included Batha, Tufstalt, Sawmill and Moulay Boua. The samples were subject to availability.

\subsection{Instrumentation}

A questionnaire, including icons and images drawn from 26 US academic websites was distributed via the Internet in three languages including English, French and Arabic. The questionnaire contained three sections. Part I introduced the study and asked for user demographic information. This section contained 18 icons and images representative of functions, actions, activities, and information types commonly seen in online learning environments on US academic websites. The questionnaire asked participants to supply the meaning for each icon or image by typing their response in the adjoining text field. Part II of the questionnaire required selecting from three images which one best represented a specific function or idea. The functions represented included the following: Internet chat, group discussion online, submitting an assignment online, and accessing a library. An additional question presented three images of professors in different clothes and backgrounds and asked the user to select which professor they would prefer to take a course from and why. Additional comment fields were provided at the end of each section.

\subsection{Procedure}

The questionnaire was distributed via the Internet in the US and Morocco during the Fall Term of 2004 and in Sri Lanka during the fall of 2005. Participants who were fluent in English filled the form out directly on the Internet; the other participants filled out the printed version in their preferred language while looking at the English version on the screen.

Two types of qualitative analysis were conducted to assess the effectiveness of the icons in conveying the intended meanings and to examine the participant's interpretation of the icon as a representation of a function or object or as a visual representation only. The first method involved a content analysis conducted in each of the three cultural contexts to derive categories from the meanings assigned to the 18 icons. From the content analysis, the interpretations were sorted into a range that included the responses "Closest to the Intended Meaning", "Similar to the Intended Meaning", and "Less Related to the Intended Meaning". This analysis associated the range of interpretations produced by each icon with the categories of relatedness that indicated the ability of the graphic image to convey a predictable message. Subsequent analysis examined the interpretations in terms of their literal versus interpretive emphasis and whether the meanings were computer related or of a general nature. Analysis of the individual image selections included identification of preference patterns and dominant themes that emerged within interpretations of the cultural groups. 


\section{Icons that describe a function}

Of the 18 icons included in the questionnaire, 12 were classified as icons that describe functions. Because they describe functions, these icons tend to be common and frequently used.

When the 18 icon interpretations from the three countries were analysed according to their closeness to the intended meanings within the groups, $53 \%$ of the US interpretations were "Closest to the Intended Meaning". Sri Lankan interpretations that were "Closest to the Intended Meaning" comprised 44\%, with $36 \%$ of the interpretations from Moroccans closely related. Since US designers created the icons in North America, it is not surprising that the majority of the US responses were closely related.

Table 1: $\quad$ Functional Icons.

\begin{tabular}{|c|c|c|c|}
\hline Icon & Function & Icon & Function \\
\hline $1 \longrightarrow$ & Access Email & 2 & Search or Find \\
\hline 4 & Access Planner & 5 & Access Calendar \\
\hline 6 & $\begin{array}{l}\text { Email or Call } \\
\text { Technical Support }\end{array}$ & 7 & Chat Assistance \\
\hline 11 & Access Quiz & & $\begin{array}{l}\text { Email Reference } \\
\text { Desk }\end{array}$ \\
\hline 13 & $\begin{array}{l}\text { Access Library } \\
\text { Research Service }\end{array}$ & 14 & Enter Chat \\
\hline 15 & Network & & Return Home \\
\hline
\end{tabular}

\subsection{Accessing email}

Analysis of the Email Icon \#1, revealed that within the US and Sri Lankan responses the descriptive variations were fewer and more similar than among those provided by Moroccan participants. Among US participants and Sri Lankan, the majority of interpretations were close to the intended meanings, including responses such as "mail", "electronic mail", "sending mail", etc. The Moroccan participants from Ifrane who represent the upper class, university educated, and those most familiar with the Internet provided a majority of the closest to intended meaning responses given by this group. Although daily Internet use is less frequent, $31 \%$ among the Moroccans as a group, of the 12 closely related interpretations, all but one came from daily Internet users, suggesting that the lower percentage of accurate interpretations was due to lack of exposure to this particular online function. The majority, of Moroccan interpretations were related, but less closely so, including associated responses 
such as "message box", "things that refer to a message", etc. Although the Moroccan interpretations were predominantly web based most were not clearly enough related to be very helpful in aiding their Internet use. The Sri Lankan responses were more consistently web based and also demonstrated greater understanding of intended meanings. This greater awareness of the icon in relation to the web was associated with more accurate interpretations and more frequent Internet use. The US interpretations were predominantly web based with only ten literal interpretations given; the infrequent use among these ten US participants (once a month) suggests the level of previous exposure to the Internet determines the accuracy of understanding, even among those users who share the cultural biases of the designer.

\subsection{Enter chat}

For the Chat Icon \#14, which is a WebCT icon, the Moroccan interpretations were more closely related to the intended meaning than either of the other groups. Moroccan interpretations were more likely to stress the use of text associated with the chat function than the other groups, which indicates an emphasis on the procedural aspects of this online practice. Most families in Morocco do not have computers at home, and the multitude of cyber cafes throughout the cities where people engage in online chat may explain the higher percentage of Moroccan participants who understood this icon. The majority of the Moroccan interpretations were web related with most from daily Internet users. Among the closely related Sri Lankan responses, 92\% were from daily Internet users and $100 \%$ of the closely related US responses were from daily users. The US and Sri Lankan participants gave less related and more general interpretations and these tended to be from the participants who used the Internet on a less frequent basis.

\subsection{Network}

The Network Icon \#15 elicited relatively predictable interpretations across all countries. The majority of closely related responses interpreted the icon as a representation of a "network", "a connection to the Internet", or "two interconnected computers". There range of descriptive variations was greater within the closely related US and Moroccan responses than within the closely related Sri Lankan interpretations. Among the Moroccans and Sri Lankan interpretations in this category, $45 \%$ were daily Internet users. Slightly more of the closely related US interpretations were from were daily Internet users. Interpretations of the Network Icon were predominantly web related for all three countries. Nearly all the responses given were web based with the majority from daily Internet users. These responses suggest that frequency of use was a salient factor in determining the users understanding or misinterpretation of the icon.

\subsection{Calendar}

All the countries interpreted the Calendar Icon \#5 with considerable accuracy. The US had more closely related responses, followed by Sri Lanka and Morocco. 
Within the Moroccan responses, 34\% were daily users, indicating that even without the benefit of very frequent exposure, the majority understood this icon. Among the US interpretations, $95 \%$ were web based with $75 \%$ of these from daily Internet users. Among the Moroccan interpretations, most were web-based with $60 \%$ from daily Internet users where as $87 \%$ of the Sri Lankan responses were web based with $51 \%$ of these from daily Internet users. One of the less related responses given by a Sri Lankan participant identified the calendar as a representation of a temporary house. It is important to note that a large number of tents were put up in the coastal areas of Sri Lanka after the tsunami incident in 2005. The aftermath of the tsunami may have influenced the participant in identifying the slanting shape of the calendar as a tent. Another participant interpreted the meaning of the icon as "open" which may have been related to the way the calendar was depicted as a 3 dimensional object.

\subsection{Home}

Analysis of the Home Icon \#17 indicated most of the participants identified the icon as representing the home page. More closely related interpretations were given by US and Sri Lankan participants and these were fewer in variation and more similar, than those provided by the Moroccan participants. Of the closely related Moroccan interpretations, the majority were from Ifrane, and represent the university educated and those most familiar with the Internet. Of this majority, $72 \%$ were daily Internet users, suggesting the meaning given to the icon depended on the participant's familiarity with the Internet. The majority (74\%) of the US interpretations and the Sri Lankan $(76 \%)$ were related to the web. US web related interpretations were comprised of $81 \%$ daily users, and among Sri Lankan web related interpretations, 38\% were from daily Internet users. Eight of the participants from Sri Lanka gave a literal interpretation, "house". Those who provided literal meanings varied in terms of their Internet use, but only two were daily users. A possible explanation would be the use of English as a second language by these participants who did not distinguish between the words "house" and "home". This icon's similarity to the thatched roof houses in Sri Lanka may have provided a sociocultural context for the single participant who interpreted the icon as representing a village.

\section{Icons that describe a kind of information}

Of the 18 icons, 6 were categorized as icons that described a kind of information. These icons describe the type of information the user will access by clicking on the icon such as a campus map, information related to accessibility services, etc.

\subsection{Accessibility services}

The Accessibility Icon's image is internationally used for public information. As an icon, it conveyed a fairly predicable meaning with $80 \%$ of US interpretations, $58 \%$ of Moroccans and $59 \%$ of the Sri Lankan Closest to the Intended Meaning. 
Table 2: $\quad$ Information type icons.

\begin{tabular}{|l|l|l|l|}
\hline Icon & Information Type & Icon & Information Type \\
\hline & $\begin{array}{l}\text { Community } \\
\text { Information }\end{array}$ & $\begin{array}{l}\text { Accessibility } \\
\text { Services }\end{array}$ \\
\hline
\end{tabular}

\subsection{Campus map}

Analysis of the Campus Map Icon \#4 indicated 64\% of US interpretations were coded "Closest to the Intended Meaning" as were 37\% of Sri Lankan and 17\% of the Moroccan interpretations.

Table 3: $\quad$ Individual icon and image options.

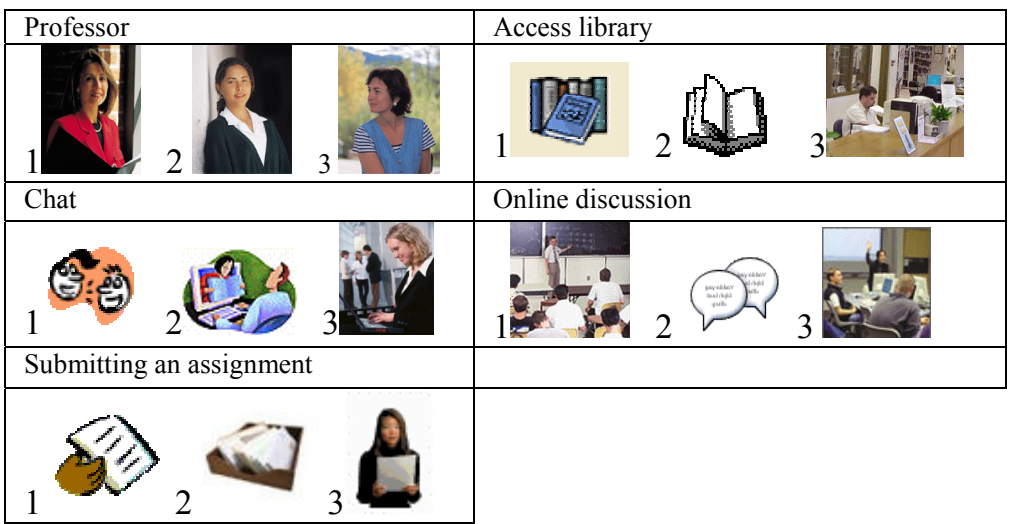

\section{Individual preferences}

Analysis of the professor picture preferences indicated picture \#1, the most formally dressed professor, was preferred by 53\% of Sri Lankan and 51\% of Moroccan participants, but only by $31 \%$ of US participants. Comments from the Moroccan participants regarding picture \#1 exemplify themes that emerged. Professionalism: "The professor in Picture \#1 seems really professional". Seriousness and Respect: "She looks like a person that we respect". Appearance is Key: "According to her appearance, she seems like a professor who could teach". The following interpretation from the Moroccan researchers elaborates the theme of professionalism and what Hofstede [5] identified as the Power Distance Cultural Dimension: "All the comments related to the person in photograph \#1 as professional indicate an important value in Moroccan society 
related to the expectation that a teacher looks professional. The professor is seen almost as a God and is invested with a great deal of respect. Especially with the older generations or those unfamiliar with more western countries and cultures, jeans are associated with construction workers, etc. Even among the younger generations people are still do not question the values and principles taught to them by their parents and teachers."

The most casually dressed professor \#3 was preferred by $47 \%$, the majority, of the US participants.

\subsection{Image preferences in representing online functions}

Image \#1, a WebCT Chat Icon, was the preferred representation of the function "Chat" by $48 \%$ of Moroccan and $64 \%$ of US participants. Sri Lankan participants chose picture \#2. Picture \#1 was the preferred image to represent "Submitting an Assignment" by $43 \%$ of the Moroccan, $81 \%$ of the US, and $76 \%$ of the Sri Lankan participants. Picture \#3 was preferred to represent "Group Discussion Online" by more than half of the Moroccan, Sri Lankan and US participants. Picture \#1, an illustration, was the image preference for representing "Accessing a Library" by $76 \%$ of US participants, $67 \%$ of Moroccan and $81 \%$ of Sri Lankan participants. The majority of preferred images were illustrations or cartoons; only one preferred image was a photograph.

\section{Conclusions and implications for design}

Evidence from this study supports the hypothesis that frequency of Internet use determines the ability to interpret the intended meanings of the icons and images. Greater awareness of the icon in relation to its function on the Internet was repeatedly associated with more frequent Internet use and with interpretations more closely related to the intended meaning of the icon. Conversely, more general interpretations tended to be associated with less frequent Internet use. Even among US participant's who share the cultural biases of the designer, those who used the Internet less frequently tended to give less closely related responses. Culturally related interpretations were more frequently associated with less related and literal meanings from participant's who had less frequent exposure to the Internet. In general, Functional Icons elicited responses more closely related to the intended meanings than Information Type Icons. The Accessibility Icon \# 10, which elicited fairly accurate interpretations, was the exception. This exception may be related to the international use of this symbol.

Icons and images that rely on literal interpretations may be most reliable in developing web materials for cross-cultural users. Images and icons, which were representational and contained little detail, were less likely to elicit unintended interpretations. Individual image preferences for online functions suggest most users preferred representations that were conceptually focused and visually simple. Icons that were photographic were least frequently selected. In selecting images of professors, teachers or staff for use in an international context, 
consideration should be given to image details that may reflect cultural differences relating to the power distance dimension.

\section{References}

[1] Tzeng O.C. S., Trung, N.T. \& Reiber, R., Cross-Cultural Comparisons on Psychosemantics of Icons and Graphics. International Journal of Psychology, 25, pp. 77-97, 1990.

[2] Preece, J., Human Computer Interaction, Addison Wesley: Wokingham, UK, 1994.

[3] Jonesson, D., Maurer, H. \& Goldman-Segall, R, DynamIcons as dynamic graphic interfaces: Interpreting the meaning of visual representation. Intelligent Tutoring Systems, 12(1), pp.35-48, 1996.

[4] Evers, V., Cross-Cultural Understanding of Icon Design: A Cross-Cultural Analysis of Icon Recognition. Proceedings of the International Workshop on Internationalisation of Products and Systems, eds. E. del Galdo, G. Prahbu, IWIPS: Rochester, NY, 1999.

[5] Hofstede, G., Cultures and Organizations: Software of the Mind, McGraw-Hill: New York, 1991.

[6] Fernandes, T., Global Interface Design, Academic Press: London, 1995.

[7] Yeo, A., Cultural User Interfaces: A Silver Lining in Cultural Diversity. SIGCHI bulletin, 28(3), pp.4-6, 1996.

[8] Duncker, E., Cross-Cultural Usability of the Library Metaphor. International Proceedings of the Second ACM/IEEE-CS Joint Conference on DigitalLibraries 2002, Portland, Oregon, pp. 223-230, 2002.

[9] Shaw, J.B. \& Barett-Power, E., The Effects of Diversity on Small Work Group Processes and Performances. Human Relations, 51(10), pp.1307$1325,1998$. 